\title{
A Modern Framework of Adolescence Health Programs
}

\author{
Francesca Mastorci ${ }^{1}$, Alessandro Vienna ${ }^{2}$, Alessandro Pingitore ${ }^{1}$ \\ ${ }^{1}$ Clinical Physiology Institute, Consiglio Nazionale delle Ricerche Area della Ricerca di Pisa (CNR), Pisa, Italy \\ ${ }^{2}$ Ministero dell'Istruzione, Dipartimento per il sistema educativo di istruzione e di formazione, Direzione generale per lo studente, \\ l'inclusione e l'orientamento scolastico, Rome, Italy \\ Email: pingi@ifc.cnr.it
}

How to cite this paper: Mastorci, F., Vienna, A. and Pingitore, A. (2021) A Modern Framework of Adolescence Health Programs. Health, 13, 685-693.

https://doi.org/10.4236/health.2021.137052

Received: April 14, 2021

Accepted: July 9, 2021

Published: July 12, 2021

Copyright (c) 2021 by author(s) and Scientific Research Publishing Inc. This work is licensed under the Creative Commons Attribution International License (CC BY 4.0).

http://creativecommons.org/licenses/by/4.0/ (c) (i) Open Access

\begin{abstract}
Adolescence represents a sensitive time period during an individual's development during which physical, psychological, and social risk factors for non-communicable diseases are established. As such, there is a necessity to engage adolescents in planning and decision-making processes that will affect their future health. This paper describes the need for more innovative and transversal actions, starting from the utility of a multidisciplinary and systemic approach to health. One way in which this can be achieved is by shifting adolescent health programs (AHPs) from a clinically oriented to an education-based model. In particular, AHPs need to consider a personalized and systemic approach to the well-being of adolescents in order to potentiate their strengths and improve weaknesses, enhancing self-esteem, empowerment, and resilience. This is in line with the complexity of human physiology and psychology, as well as scientific evidence showing that several factors significantly influence health status including lifestyle, social context, emotional experiences, and cognitive skills, as demonstrated by the AVATAR Project ("A new purpose for promotion and eVAluation of healTh and well-being Among healthy teenageRs"). This project-which provides a new framework for AHPs-was carried out in Italy and enrolled healthy students as subjects, and aimed to promote well-being in adolescents using a systemic and multistakeholder approach that involved creating a network composed of the different figures and environments around adolescents and moving toward a more school-based and student-centered approach to adolescent health education.
\end{abstract}

\section{Keywords}

Adolescent, Health, Well-Being, School, Adolescent Health Program, Prevention 


\section{Introduction}

Health, well-being, and quality of life are interconnected concepts with multidimensional and multidisciplinary aspects. According to the World Health Organization (WHO), health is a dynamic state characterized by physical, mental, and social potential that meets the demands of life commensurate with age, culture, and personal responsibility [1]. Well-being is defined as a condition of dynamic equilibrium or physiological homeostasis between personality and life events or challenges, while quality of life is an overarching notion that is affected in a complex way by an individual's physical health, psychological state, personal beliefs, and social relationships. These definitions match that of complex systems such as human beings, defined as a network of cooperating units (i.e., systems) characterized by global properties independent of the detailed features of the units in the absence of cooperation [2].

Taken as a whole, these concepts highlight the utility of approaching health from a multidisciplinary, integrated, and personalized perspective. This has even greater importance during a sensitive time window such as adolescence, which is characterized by drastic physical, psychological, and social changes as well as by conflicts between instinct and reasoning and between impulse and thought [3]. In this context-which is highly variable across subjects-it is critical to determine the perception that adolescents have of their own health and well-being. Components have been specified for three reasons: 1) ease of use when formatting individual papers, 2) automatic compliance to electronic requirements that facilitate the concurrent or later production of electronic products, and 3) conformity of style throughout a journal paper. Margins, column widths, line spacing, and type styles are built-in; examples of the type styles are provided throughout this document and are identified in italic type, within parentheses, following the example. Some components, such as multi-leveled equations, graphics, and tables are not prescribed, although the various table text styles are provided. The formatter will need to create these components, incorporating the applicable criteria that follow.

\section{Adolescent Health Programs (AHPs)}

Although adolescence is considered a healthy age, it also represents a period during which behavioral risk factors for non-communicable diseases are established. In response to environmental, social, and internal inputs, adolescents may experience a wide range of emotional responses according to an all-or-nothing principle, possibly as a result of ongoing brain development. This phenomenon-known as pruning-is on the one hand responsible for the imbalance in the integration between the main neural systems associated with risk behaviors (e.g. substance use or abuse and addiction behaviour); and on the other, for the development of diverse skills such as reasoning and moral evaluation as well as the capacity for abstract thinking and rational judgment. Maturation of the prefrontal cortex and its medial and ventral areas is delayed in adolescents. This 
phenomenon affects behavior, making teens more vulnerable to choices that have greater value in the short term. Therefore, a neurobiological cause justifies the attraction of adolescents to discovery of immediate emotions and pleasures. In this field, prospective studies demonstrated that adolescents with recent negative life events, such as parental loss, chronic stress, social isolation, or low quality of life, increased levels and susceptibility of substance use and abuse [4].

In this context, it is important to understand how adolescents perceive their own health and well-being in order to facilitate a healthy and smooth transition to adulthood. In line with these needs, the Lancet Commission on adolescent welfare issues recently recommended the improvement of evidenced-based strategies and policies to reduce the burden of adolescent mortality and morbidity worldwide [5]. In terms of social context, school is the environment where adolescents spend the most time, and should therefore be the principal setting for the development of health programs as it can potentially reach the vast majority of adolescents. As such, in recent years school-based interventions have become a focus of the WHO for defining health policies, healthy environments, health education, and healthy social context.

The process of learning and empowerment can be incorporated into personalized, integrated, and multidimensional AHPs that embrace physical, mental, social, and emotional aspects as well as socio-cultural environment. In this frame, school offers a sustained and efficient place to design AHP; it can be considered the optimal setting to apply and develop programs to promote health and well-being for its safety, cost-effectiveness, and flexibility. Furthermore, in the school the continuous relationship between students and teachers guarantees both trust and accessibility toward the programs of health and well-being promotion.

Accordingly, a recent joint document released by the Italian Ministries of Health and Instruction recommended a change in adolescent health education paradigms from a thematic or sectorial approach to a global or systemic one, highlighting the need to integrate different and transverse types of intervention [6]. This is in line with the complexity of human physiology and psychology and with scientific evidence showing that several factors influence health status including lifestyle, social context, emotional experiences, and cognitive skills [7]. Another key point that should be considered in adolescence is sex differences, because menarche during this period significantly alters both the body and mind in girls. Our group recently demonstrated that menarche affects all components of life quality from mood and self-esteem to social relationships, underscoring how hormonal variations contribute to psychological and emotional changes [8].

The application of a personalized and systemic approach to the well-being of adolescents has the benefit of potentiating their strengths and improving their weaknesses, thereby enhancing self-esteem, empowerment, and resilience. It is worth noting that the definition of resilience-that is, the ability to prevent, minimize, or overcome the damaging effects of adversity-conceptually matches that of hormesis, which is included in the definition of well-being and refers to 
all physiological mechanisms that maintain equilibrium across all human systems and organs. In other words, resilience and hormesis maintain psychological and physiological equilibrium, respectively, and both are necessary to preserve global health [9]. Besides this psychophysiological network and consistent with the multidisciplinary nature of the proposed AHP framework, there should be a parallel network composed of figures who have contact with adolescents including family, teachers, and research and institutional stakeholders. In this scenario, the school-which is defined by the WHO as a "setting for health" and "context of socialization"- has a central role in promoting AHPs, as it represents an interactive social context in which adolescents can share life experiences with and thus learn from their peers.

In line with the abovementioned need, the link between research institutions and schools is an essential component of the AHP framework. Research provides an opportunity to apply an evidence-based methodology to AHP design based on systematically acquired data, allowing the development of projects and strategies focused on the needs of the educational institution, classroom, and individual students. Furthermore, systematic and longitudinal data collection makes it possible to assess the effectiveness and efficacy of applied programs and interventions. A registry comprising data for a large number of adolescents can be used by institutional stakeholders for the development of national and international health and social policies, and can facilitate the design and promotion of preventive programs that aim to maintain health by mitigating risk factors before the emergence of diseases [10]. Such primordial prevention efforts have 3 main components: a healthy diet, engagement in physical activity, and avoidance of smoking, in accordance with optimal cardiovascular health programs. Several studies on adolescents have demonstrated the effectiveness of health programs in preventing dyslipidemia, hypertension, and metabolic syndrome. However, this is insufficient for ensuring the healthy development of adolescents. In view of a larger systemic approach, the next challenge for AHPs is to incorporate variables in the psychosocial, emotional, cognitive, and lifestyle dimensions into an interactive and integrated model, assuming that these are not independent of each other [11]. This allows assessment of the contribution of individual items to a personalized well-being index, and identification of those that must be reinforced or changed through application of a personalized and focused AHP. For example, teachers can apply personalized interventions and educational programs and through longitudinal monitoring, assess their effectiveness as well as students' compliance with the program. This can also reveal new aspects of the child to his/her family, leading to a dialogue with teachers based on objective data and the implementation of the same AHP at home and at school. Furthermore, health questionnaires should be available on a web-based platform where data is automatically analyzed and made available in real time to teachers, and then archived. This type of data registry on adolescent health can guide the establishment of evidenced-based health education policies, thus strengthening the collaboration between schools, institutional stakeholders, and researchers. 


\section{The AVATAR Approach}

According to the document published by the Italian Ministries of Health and Instruction in which the need for a "global school approach" is highlighted as part of educational training, a collaboration between a research institute, Clinical Physiology Institute, and the Rete Ulisse (a National Network of Schools) has emerged within the AVATAR ("A new purpose for promotion and eVAluation of healTh and well-being Among healthy teenageRs") Project. The target of this project is the healthy adolescent. The importance of healthcare and improvement of well-being in a healthy population is in line with the concept of primordial prevention to reduce the risk of chronic or degenerative diseases in adulthood [12].

AVATAR aims to promote well-being in adolescents through a systemic and multi-stakeholder approach that involves establishing a network composed of different figures and environments surrounding the adolescent (family, school, peers, and community). Additionally, variables that encompass different dimensions of health and well-being including lifestyle, emotional state, and social context are monitored. AVATAR methodology consists of integrating information obtained from the different standardized questionnaires and translating this into user-friendly language, which is carried out by the schools using a flexible and adaptable web-based platform. This approach allows questionnaires to be completed online at school or at home, so that data can be analyzed, managed, and archived and results made available in real time. A personalized well-being index for adolescents was previously developed based on the integration of social, emotional, and cognitive skills and lifestyle [13] that allows schools to identify the strengths and weaknesses of each student, so that the former can be potentiated and the latter changed or improved through personalized educational programs. Thus, the AVATAR index intervenes at multiple levels to promote health and well-being in adolescents by combining management and empowerment and applying preventive strategies to reduce disease burden in adulthood.

In light of the need for disease prevention and in accordance with the European strategic plan for promoting the well-being of adolescents, AVATAR has the following aims: 1) on the research front, to identify factors contributing to well-being in relation to primordial prevention; and 2) in terms of teaching and school policy, to establish a model that supports autonomous planning by schools, the training of teachers, and the transfer of good practices. Collaboration between researchers and schools is a key element of the AVATAR Project, which serves a tool for defining a shared model of health education both at a national level and at the level of individual schools. In particular, AVATAR combines the accuracy and objectivity of scientific methodology with the needs that are identified by the school system in terms of health, well-being, and educational success of adolescents. The AVATAR platform processes data automatically and presents these in a comprehensible manner in real time to teachers, allowing schools to develop training and didactic strategies to enhance students' 
education, well-being, and motivation to learn and reduce risk behaviors through a systemic, integrated, and personalized approach. Since its launch, the AVATAR platform has made available to schools and the scientific community up-to-date and comparative data on the behaviors and perceptions of adolescents, creating a network of multidisciplinary collaborators focused on developing interventions for disease prevention and health promotion. In particular, the end goal of the AVATAR methodology is to strengthen the scientific approach to improving the health and well-being of adolescents through the following measures: 1) launching campaigns aimed at modifying risk behaviors in adolescents; 2) raising adolescents' awareness of being in control of their own growth trajectory; 3 ) involving institutions in the health and well-being of the adolescent population; 4) evaluating the effectiveness of actions implemented by the school; and 5) promoting the transfer of good practices for the education, training, and well-being of students.

\section{Discussion}

Adolescence is a sensitive time period of changes-including physical, psychological, hormonal, cognitive, social, and emotional-in all areas of life. Because of these rapid and unpredictable changes, adolescence should be the first target of every prevention plan both from the clinical and educational perspectives, in order to support adolescents' healthy development and promote health and well-being in adulthood. Adolescence is considered the healthiest life stage but is paradoxically marked by surges in the risk of morbidity and mortality. In this context, AHPs should be evidenced-based school health initiatives that are aligned to the real needs and priorities of adolescents, and should identify the strengths and vulnerabilities of each adolescent in order to potentiate the former and change or improve the latter through careful analysis of the major variables linked to health and well-being such as lifestyle habits, social context, and emotional and cognitive states. In addition, the school-considered as a "context for health"-should promote resilience, emotional well-being, empowerment, and awareness in adolescents according to their health and well-being profile.

The promotion of adolescent health should be the main focus of public health policies school health programs, with the aim of preventing morbidity and early mortality in adulthood. Although the commitment of various stakeholders (researchers, school, family, and community) has increased in this regard, the need for more innovative and transversal actions remains.

In this perspective, AVATAR project includes preventive intervention strategies to improve psychosocial and environmental conditions having potential health implications through the development of a network of different institutional, political and social stakeholders of health. In this way, AVATAR facilitates the development of a network among the institutional stakeholders of health to promote health preventive strategies and policies focused on the epidemiological and objective data on adolescent well-being and health. This approach 


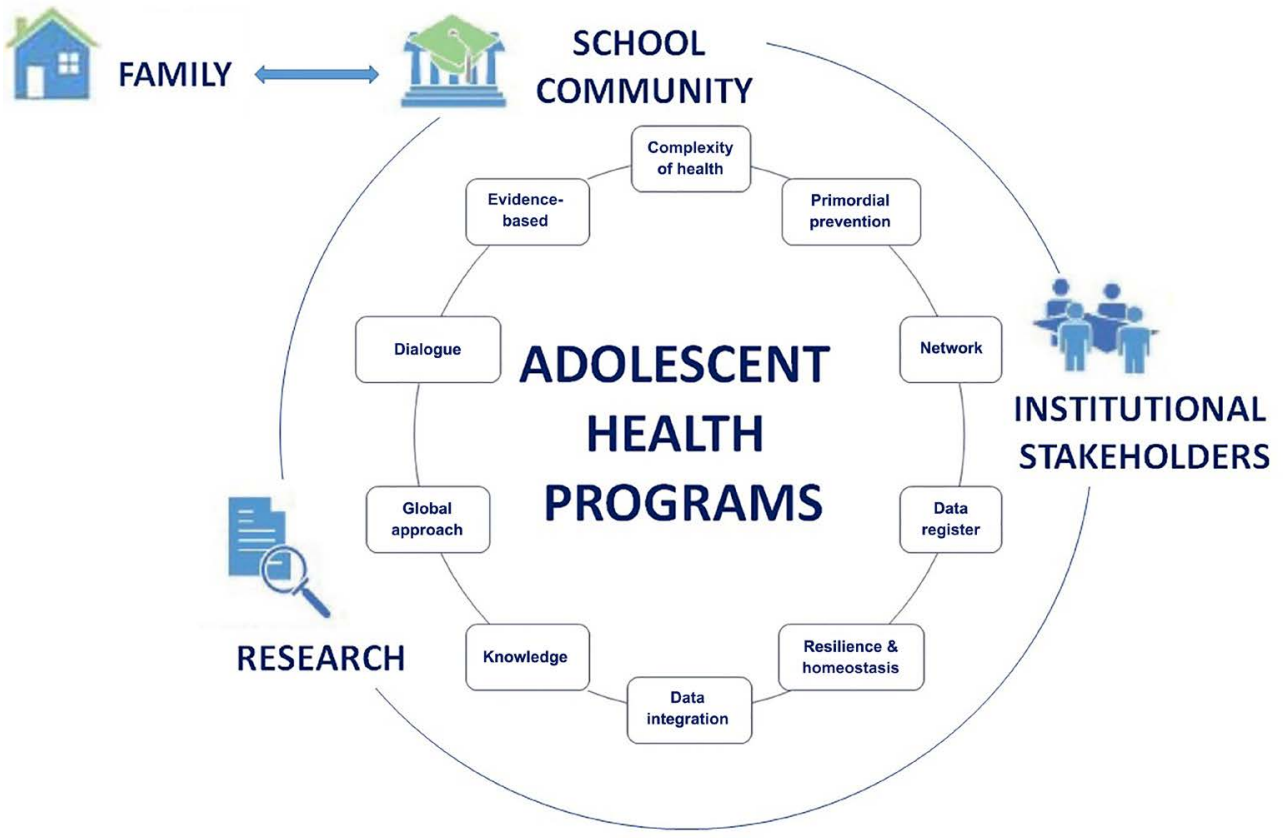

Figure 1. Adolescent health program framework. The central circle represents the keywords of the AHPs, while the stakeholders of health are represented on the outside.

would help teachers select more appropriately personalized interventions and educational programs for individual students and for the classroom, thus, monitor their compliance and effectiveness.

\section{Conclusions}

In conclusion, the educational programs should be focused to identify the strength and the fragile characteristics of each adolescent in order to potentiate the first ones and to change or improve the others. This needs the knowledge of the individual sides of the adolescent and how the single sides talk to each other. This provides the opportunity to create dedicated and personalized interventions that help adolescents increase her/his awareness, resilience and thus subjective well-being.

The biggest challenge for promotion of adolescent health involves the need to engage adolescents in planning and decision-making processes that will affect their health, now and in future, must lead to a transition from a clinically oriented system to an education-based model that considers individuals in all aspects of experience and in different social contexts. A new model of integration between components of health and well-being can be evolved to make services for disease prevention and health promotion more student-friendly, moving from health facilities to more school-based and student-centred. Thus, starting from the utility of a multidisciplinary and systemic approach to health, the following key words are proposed for the new AHP framework: complexity of health, primordial prevention, network, data registry, resilience and homeostasis, data integration, evidence-based, dialogue, and knowledge (Figure 1). 


\section{Author Contributions}

All authors listed have made a direct and intellectual contribution to the work, and have approved it for publication.

\section{Conflicts of Interest}

The authors declare no conflicts of interest regarding the publication of this paper.

\section{References}

[1] Bircher, J. (2005) Towards a Dynamic Definition of Health and Disease. Medicine, Health Care, and Philosophy, 8, 335-341. https://doi.org/10.1007/s11019-005-0538-y

[2] West, B.J., Geneston, E.L. and Grigolini, P. (2008) Maximizing Information Exchange between Complex Networks. Physics Reports, 468, 1-99. https://doi.org/10.1016/j.physrep.2008.06.003

[3] Foreword, I.G. (2019) Foreword. In: Pingitore, A., Mastorci, F. and Vassalle, C., Eds., Adolescent Health and Wellbeing. Current Strategies and Future Trends. Springer Nature Switzerland AG, Basel.

[4] Barrett, A. and Turner, R. (2006) Family Structure and Substance Use Problems in Adolescence and Early Adulthood: Examining Explanations for the Relationship. Addiction, 101, 109-120. https://doi.org/10.1111/j.1360-0443.2005.01296.x

[5] Xu, T., Tomokawa, S., Gregorio Jr, E.R., Mannava, P., Nagai, M. and Sobel, H. (2020) School-Based Interventions to Promote Adolescent Health: A Systematic Review in Low- and Middle-Income Countries of WHO Western Pacific Region. PLoS ONE, 15, e0230046. https://doi.org/10.1371/journal.pone.0230046

[6] Conferenza Stato-Regioni. Atto Rep. n.2/CSR, 17/01/2019: Italian Ministries of Health and Instruction “Integrated 'Policy' Guidelines for the School That Promotes Health", 2019.

[7] Patton, G.C., Sawyer, S.M., Santelli, J.S., Ross, D.A., Afifi, R., Allen, N.B., et al. (2016) Our Future: A Lancet Commission on Adolescent Health and Wellbeing. The Lancet, 387, 2423-2478. https://doi.org/10.1016/S0140-6736(16)00579-1

[8] Mastorci, F., Piaggi, P., Bastiani, L., Trivellini, G., Doveri, C., Casu, A., et al. (2020) The Impact of Menarche on Health-Related Quality of Life in a Sample of Italian Adolescents: Evidence from School-Based AVATAR Project. European Journal of Pediatrics, 179, 973-978. https://doi.org/10.1007/s00431-020-03594-8

[9] Stebbing, A.R. (1982) Hormesis-The Stimulation of Growth by Low Levels of Inhibitors. The Science of the Total Environment, 22, 213-234.

https://doi.org/10.1016/0048-9697(82)90066-3

[10] Weintraub, W.S., Daniels, S.R., Burke, L.E., Franklin, B.A., Goff Jr., D.C., Hayman, L.L., et al. (2011) Value of Primordial and Primary Prevention for Cardiovascular Disease: A Policy Statement from the American Heart Association. Circulation, 124, 967-990. https://doi.org/10.1161/CIR.0b013e3182285a81

[11] Mastorci, F., Bastiani, L., Trivellini, G., Doveri, C., Vassalle, C. and Pingitore, A. (2020) A New Integrated Approach for Adolescent Health and Well-Being: The AVATAR Project. Health and Quality of Life Outcomes, 18, 77. https://doi.org/10.1186/s12955-020-01291-6

[12] Trivellini, G., Doveri, C., Mastorci, F., Bastiani, L., Cappa, C., Vassalle, C., et al. 
(2018). Innovative Web-Based Tool for Promoting Well-Being among Healthy Adolescents: An Implementation Protocol. Journal of Translational Science, 5, 1-5. https://doi.org/10.15761/JTS.1000261

[13] Mastorci, F., Bastiani, L., Doveri, C., Trivellini, G., Casu, A., Vassalle, C., et al. (2020) Adolescent Health: A Framework for Developing an Innovative Personalized Well-Being Index. Frontiers in Pediatrics, 8, 181.

https://doi.org/10.3389/fped.2020.00181 